\title{
Materiales de aleación aluminio-silicio aplicados en la fabricación de partes de motores de combustión interna alternativos Parte I
}

\section{Aluminum-silicon alloy materials applied in the manufacture of parts of reciprocating internal combustion engines Part I}

Barona López Gustavo. ${ }^{1}$ Luis Efraín Velasteguí. ${ }^{2}$

\begin{abstract}
.
DOI: https://doi.org/10.33262/cienciadigital.v3i3.2.758

This paper presents a systematic knowledge of the chemical composition, type of molding and treatment, mechanical and thermal properties, of aluminium-silicon (Al-Si) alloys applied in the manufacture of structural parts of reciprocating internal combustion engines (RICE), with the aim of providing support for the development of researches when compare, analyze or select the presented alloys. For which, a first paper is presented, which indicates, the structural parts of these types of engines are manufactured with Al-Si alloys. The paper begins with concepts, characteristics and classification of RICE. Subsequently, the characteristics, function of the parts, work cycles and other aspects of the Otto four- and two-stroke RICE are described, in addition to the Al-Si alloy cylinder block, and the structural parts of Otto engines made of Al-Si alloys are shown. To deepen the issue, the characteristics and the duty cycle of diesel engines have been written. Finally, a summary of characteristics of the Diesel and Otto engine was prepared. The paper shows as a result a systematization of the subjects investigated where Al-Si alloys integrate the structural parts of the RICE used in different industrial applications.
\end{abstract}

Keywords: aluminium-silicon alloys, reciprocating internal combustion engines, Al-Si alloy cylinder block, Otto engine, Diesel engine, structural parts engine.

\section{Resumen.}

En este artículo se presenta un conocimiento sistematizado de la composición química, tipo de moldeo y tratamiento, propiedades mecánicas y térmicas, de las aleaciones aluminio-silicio (Al-Si) aplicadas en la fabricación de partes estructurales de los motores de combustión interna alternativos (MCIA), con el objetivo de proporcionar un fundamento para el desarrollo de investigaciones al comparar, analizar o seleccionar las aleaciones presentadas. Para lo cual, se presenta un primer artículo que da a conocer que partes estructurales de estos tipos de motores son fabricados con aleaciones de Al-Si. El articulo comienza con conceptos, características y clasificación de los MCIA. Posteriormente se describen

\footnotetext{
${ }^{1}$ Escuela Politécnica Nacional, Facultad de Ingeniería Mecánica, Quito, Ecuador, barona_gustavo@ hotmail.com

2 Editorial Ciencia Digital, Ambato, Ecuador, luisefrainvelastegui@cienciadigital.org
} 
las características, función de las partes, ciclos de trabajo y otros aspectos más, de los MCIA Otto de cuatro y dos tiempos, también se hace referencia al block de cilindros de aleación Al-Si, y se muestran las partes estructurales de los motores Otto fabricadas con aleaciones de Al-Si. Para profundizar el tema se ha redactado las características y el ciclo de trabajo de los motores Diésel. Para finalizar, se elaboró un resumen de características del motor Diésel y del motor Otto. El artículo muestra como resultado una sistematización de los temas investigados donde se relaciona las aleaciones de Al-Si con las partes estructurales de los MCIA utilizados en diferentes aplicaciones industriales.

Palabras clave: Aleaciones aluminio-silicio, motor de combustión interna alternativo, bloque de cilindros, motor Otto y Diésel, partes estructurales del motor.

\section{INTRODUCCIÓN}

La historia relata que el motor de combustión interna se desarrolló a través de la evolución de la máquina de vapor, que es nada menos que un motor de combustión externa. Varias ideas fueron aportadas por nombres como Lebon y Rivaza, hasta que, en el año de 1852, el francés de origen belga, Ettiene Lenoir, construyó un motor de combustión interna de dos tiempos de baja potencia. Posteriormente, en 1862, Beau de Rochas ideó un ciclo de cuatro tiempos que fue desarrollado por el ingeniero alemán Nikolaus August Otto, quien, en el año de 1876, construyó un motor de combustión interna que consumía gas de alumbrado. En su honor este motor se denomina motor Otto de cuatro tiempos que sirvió para el desarrollo y fabricación de nuevos motores (Sanz, 2007) (Villegas et al., 2007).

Entre los colaboradores de Otto se encontraba Gottlieb Daimler, quien construyó un motor alimentado a base de gasolina, pero con el principio de funcionamiento del motor Otto, lo cual sirvió, para que Daimler en compañía de Wilhelm Maybach, en el año de 1886, ensayaran el motor de combustión interna a gasolina y lo montaran en un carruaje de cuatro ruedas. En ese mismo año, el ingeniero alemán, Karl Benz construyó un motor de combustión interna a gasolina de cuatro tiempos, con potencia de $3 / 4 \mathrm{HP}$, y lo monto en una carrocería tubular de tres ruedas, esta invención es considerada como el primer vehículo fabricado de la historia (Sanz, 2007) (Villegas et al., 2007). La Figura 1 muestra el automóvil de tres ruedas fabricado por Karl Benz con MCIA de cuatro tiempos.

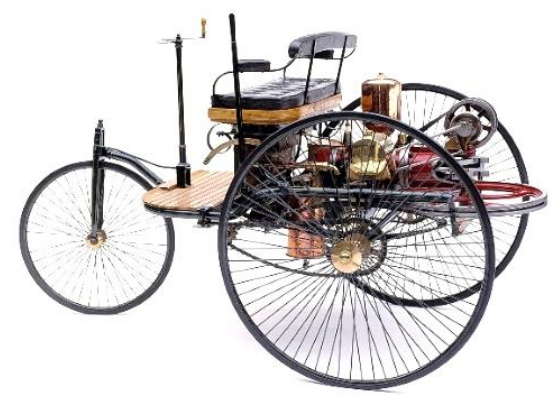

Figura 1. Vehículo de tres ruedas fabricado por Karl Benz con motor de combustión interna (NOSOLOINGENIERIA, 2013).

Los ingenieros galos René Panhard y Emile Levassor compraron la patente de Daimler, quienes entre los años de 1890 a 1891 realizaron ensayos, y fundaron la primera empresa de automóviles del mundo Panhard-Levassor (Sanz, 2007) (Villegas et al., 2007). 
Con el objetivo de superar la eficiencia del motor Otto, el ingeniero francés Rudolf Diésel, en el año de 1897, construyo la máquina térmica más eficiente de todos los tiempos, el famoso motor Diésel. Este motor de combustión interna de 4 tiempos funcionó con combustibles pesados y no necesitaba sistema de encendido por chispa, tenía una potencia aproximada de 25 HP. Rudolf Diésel murió en el año 1913, pero después de su muerte, en el año de1924, fue montado por primera vez el motor Diésel en un camión de la marca MAN (Sanz, 2007) (Villegas et al., 2007), como se muestra en la Figura 2, siendo el primer camión constituido con un motor diésel de inyección directa. Este tipo de motor debido a su fabricación robusta, en comparación a un motor a gasolina, se lo utilizó en motores estacionarios, máquinas agrícolas, camiones y maquinaria pesada.

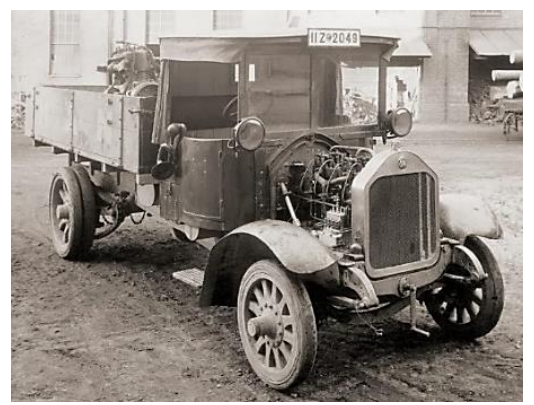

Figura 2. Camión MAN con motor diésel de inyección directa (pressreader, 2015).

En la historia existe el nombre del ingeniero alemán Félix Wankel, quien ideó otro tipo de motor de combustión interna de cuatro tiempos, el motor rotativo Wankel. El primer motor rotativo funcional se construyó en el año de 1957, y posteriormente en 1967 se fabrica un automóvil con motor Wankel sin mucho éxito hasta ahora. El motor Wankel técnicamente se define como un motor de combustión interna rotativo (MCIR), ya que su trabajo se obtiene mediante el desplazamiento rotativo de un embolo. Actualmente siguen gobernando los motores de combustión interna ideados por Otto y Diésel (Sanz, 2007) (Villegas et al., 2007) (Rovira y Muñoz, 2015).

El desarrollo de toda clase de vehículos con motor Otto o Diésel se debe al esfuerzo, investigación, diseño y construcción, por parte de un sin número de investigadores. Sin embargo, la industria automotriz, naval, aeronáutica y militar, demandan de motores más eficientes, por lo que, se tendrá que desarrollar nuevas aleaciones, rediseñar el motor, innovar materiales, mejorar los sistemas de inyección electrónica, y otras alternativas que deberán ser propuestas por las industrias de manufactura.

El motor Otto técnicamente se define como un motor de combustión interna alternativo (MCIA), ya que su trabajo se obtiene mediante el desplazamiento lineal de un émbolo (Rovira y Muñoz, 2015).

Los vehículos con MCIA Otto y Diésel representan aprox. el 95\% del total de ventas en el mundo, mientras que, el 5\% son ventas de vehículos eléctricos e híbridos. Una de las razones actuales para que el vehículo eléctrico no sea la solución medioambiental, se debe a que su motor necesita de imanes permanentes que son fabricado a partir del neodimio y disprosio, metales que son obtenidos a partir de tierras raras. Estas tierras son escasas, su aislación para obtener los metales arroja desechos tóxicos, además, los metales son de 
difícil reciclaje, lo que encarece los costos de fabricación. Todos estos factores negativos de los vehículos eléctricos son igual de adversos que la producción y consumo de combustibles fósiles. Además, hay que tomar en cuenta que para la fabricación de las baterías eléctricas se necesita de litio y grafeno, lo que aumenta el extractivismo.

El MCIA es el componente fundamental de un vehículo sus partes principales, como el block de cilindros, camisas de cilindros, cabezotes y pistones, son fabricadas con aleaciones de alta densidad como fundiciones grises o hierros dúctiles, o de baja densidad como Al-Si. De estas, las aleaciones de Al-Si son materiales que pueden ser innovados, ya que por su ligereza existe una reducción en el consumo de combustible y de las reducciones de gases contaminantes, por tal motivo, en la presente investigación se expondrá sus características y aplicaciones.

\section{MOTORES DE COMBUSTIÓN INTERNA}

Los motores de combustión interna alternativos (MCIA) son utilizados en aplicaciones automotrices, militares y navales, debido a las características de adaptación que presentan, sin embargo, tienen algunas aplicaciones aeronáuticas. La clasificación de los motores de combustión interna puede ser de acuerdo al movimiento que realizan sus elementos mecánicos, clasificándose en alternativos y rotativos. En este artículo se describirá los MCIA.

\subsection{Motor de combustión interna alternativo}

El motor de combustión interna alternativo (MCIA) es un tipo de máquina térmica que transforma la energía química, originada por la reacción de un combustible en presencia de comburente al interior de una cámara de combustión, en energía mecánica, que es aprovechada para realizar trabajo mecánico (Sanz, 2007) (Rovira y Muñoz, 2015).

Su nombre de combustión interna se debe a que la reacción de tipo explosión se produce en el interior de la máquina (en el block o bloque de cilindros) que está cerrada herméticamente por un cabezote o culata. En cambio, el termino alternativo se debe a que el trabajo mecánico se obtiene mediante el desplazamiento lineal del émbolo (pistón) sustentado en un mecanismo biela-manivela (biela-cigüeñal), el termino alternativo también es equivalente a reciprocante. (Sanz, 2007) (Rovira y Muñoz, 2015) (Kumar, 2019). La Figura 3 muestra un esquema básico de los principales componentes estructurales del MCIA.

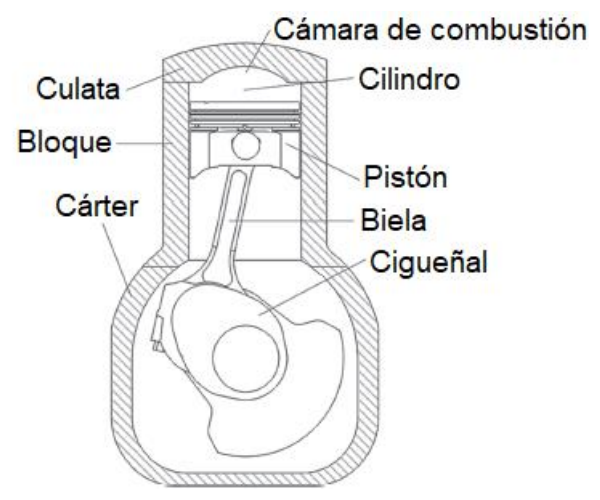

Figura 3. Componentes estructurales del MCIA (Rovira y Muñoz, 2015). 
Durante la redacción del artículo la denominación técnica de motor de combustión interna alternativo podrá ser simplemente escrito como motor de combustión interna u otro sinónimo, excluido la palabra alternativo, con el fin de simplificar términos.

\subsection{Características de los motores de combustión interna alternativos}

Los motores de combustión interna alternativos que son utilizados en la industria militar, naval, automotriz, y en algunos casos en la aeronáutica. Estos motores deben ser altamente eficientes para reducir al mínimo el impacto ambiental en el planeta. Por lo cual, las características principales de los MCIA son las siguientes:

- El material de partes estructurales como blocks de cilindros, camisas de cilindros, cabezote y pistones, deben ser ligeros, para reducir el consumo de combustible.

- Evitar al mínimo las pérdidas de energía producida por la combustión.

- La energía producida por la combustión debe encaminarse a tener alto rendimiento.

- Minimizar la contaminación ambiental a causa de gases de combustión.

- Elevada potencia a partir de un mínimo consumo de combustible.

- Sistemas de inyección de combustible eficientes.

- Costos de producción económicos.

- Costo de mantenimiento económicos.

\subsection{Clasificación de los motores de combustión interna alternativos}

La Figura 4 muestra que los MCIA pueden ser clasificados mediante cinco formas, las cuales son según su proceso de combustión, modo de realizar el ciclo, tipo de refrigeración, presión de admisión, número y disposición de los cilindros.

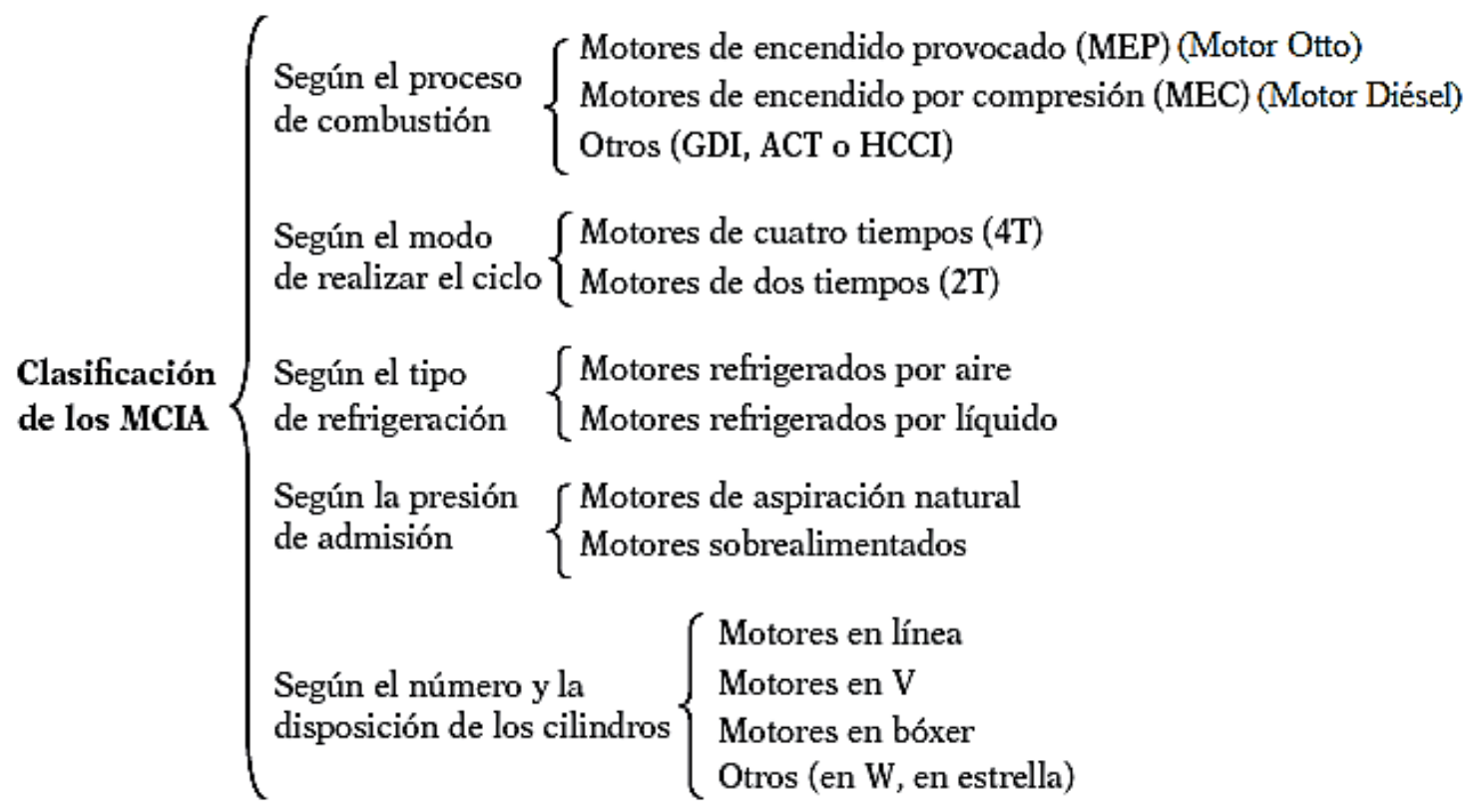

Figura 4. Clasificación de los MCIA (Rovira y Muñoz, 2015). 
Sin embargo, en la publicación elaborada por Kaumar (2019) se menciona que los motores de combustión interna pueden ser clasificados de la siguiente forma:

- Diseño básico: alternativos o rotativos.

- Tipo de ignición y combustión: ignición sea por chispa o compresión, combustión homogénea o heterogénea.

- Ciclo de trabajo: cuatro y dos tiempos.

- Proceso de admisión de aire: aspiración natural, turboalimentado, sobrealimentado.

- Localización de las válvulas: sobrecabezote, en el block y en el cabezote.

- Posición y numero de cilindros: motor mono cilíndrico, motor en línea, motor en $\mathrm{V}$, motor de cilindros opuestos, motor de pistón opuesto, motor radial.

- Refrigeración: refrigerado por aire, refrigerado por líquido, refrigerado por agua.

- Combustible, diésel, gasolina, gas, dimetil éter, etanol, metanol.

- Método de inyección del combustible: Inyección directa, inyección multipunto, al cuerpo de aceleración.

\section{MOTOR OTTO}

Las características del motor Otto radican en que su energía mecánica lo obtiene a partir de combustibles volátiles de baja viscosidad, la ignición del combustible se logra mediante una chispa, y sus partes estructurales son menos robustas que los motores Diésel. Según su ciclo de trabajo se clasifican en motores Otto de cuatro tiempos (4T) y motores Otto de dos tiempos (2T). La función de las partes principales de un motor Otto 4T es semejante a los de un motor Otto 2T, existiendo diferencias en sus ciclos de trabajo. El block de cilindros es el elemento de mayor importancia en un motor Otto, sobre el, se montan otros componentes tanto fijos y móviles, posee orificios internos llamados cilindros que pueden ser parte integral del block, o piezas desmontables que se introducen en su interior llamadas camisas. Las partes estructurales de MCIA tipo Otto fabricadas con aleaciones de Al-Si son extensamente utilizadas para vehículos de baja y mediana potencia.

\subsection{Características del motor Otto}

Reciben el nombre de motores de encendido provocado (MEP), motores de encendido por chispa, pero se debe evitar la denominación de motor de explosión, ya que en el seno del motor tiene lugar una combustión inducida por un agente, no una explosión. Generalmente utiliza combustibles como gasolina, GLP, etanol, alcoholes ligeros, gas natural, metano o gas de síntesis (Sanz, 2007) (Rovira y Muñoz, 2015).

Son motores en los que al final del proceso de compresión se dispone de una mezcla homogénea aire-combustible en estado gaseoso (o vaporizado). La combustión se produce mediante la deflagración (combustión progresiva) de la mezcla, que se propaga mediante un frente de llama, y la ignición o encendido se consigue mediante un agente externo, generalmente una chispa procedente de una bujía (Rovira y Muñoz, 2015).

El motor Otto de cuatro tiempos (4T) es utilizado en automóviles, camionetas, automóviles de competición, montacargas y vehículos de mediano cilindraje (incluye aplicaciones aeronáuticas). En cambio, el motor Otto de dos tiempos (2T) es utilizado en 
motocicletas, motonetas, embarcaciones marinas de bajo cilindraje, fumigadoras, motosierras, máquinas manuales de bajo cilindraje, y en algunos vehículos de bajo y mediano cilindraje. Tanto los motores Otto $4 \mathrm{~T}$ y $2 \mathrm{~T}$ pueden ser refrigerados mediante agua $o$ aire.

Debido al bajo y mediano cilindraje de los motores Otto, sus partes estructurales como el block de cilindros, camisas de cilindros, cabezote y pistones, son fabricados a partir de aleaciones de aluminio-silicio, las cuales poseen una resistencia mecánica, acorde a las solicitaciones mecánicas generadas por la ignición del combustible en el cilindro del block.

En cambio, motores de mediano y elevado cilindraje, como por ejemplo camionetas con un torque neto de aprox. $200 \mathrm{Nm}$ a $2000 \mathrm{rpm}$ y potencia neta de alrededor de $80 \mathrm{HP}$, camiones con un torque neto de aprox. $2400 \mathrm{Nm}$ a $1000 \mathrm{rpm}$ y potencia neta de alrededor de $500 \mathrm{HP}$, u otro vehículo con torque y potencias superiores, sus partes estructurales como el block de cilindros, camisas de cilindros y cabezote, deben ser robustas, ya que soportan solicitaciones mecánicas producidas por la autoinflamación del combustible diésel. A causa de este combustible sus partes son fabricadas a partir de aleaciones de hierro-carbono (aceros).

\subsection{Motor Otto de cuatro tiempos}

Las partes estructurales de un motor Otto 4T están conformadas por un bloque de cilindros o block de cilindros (también camisas de cilindros integrales o desmontables ubicadas al interior de los cilindros), culata, junta de culata y pistón. Existe variación en su forma y geometría, según la potencia, aplicación y marca. Por otro lado, las partes del tren alternativo como las de distribución, son fabricadas para llegar a un mismo fin en cualquier marca de motor, asimismo según la marca y aplicación, existen diferentes diseños. La Figura 5 muestra las partes principales de un motor Otto de cuatro tiempos.

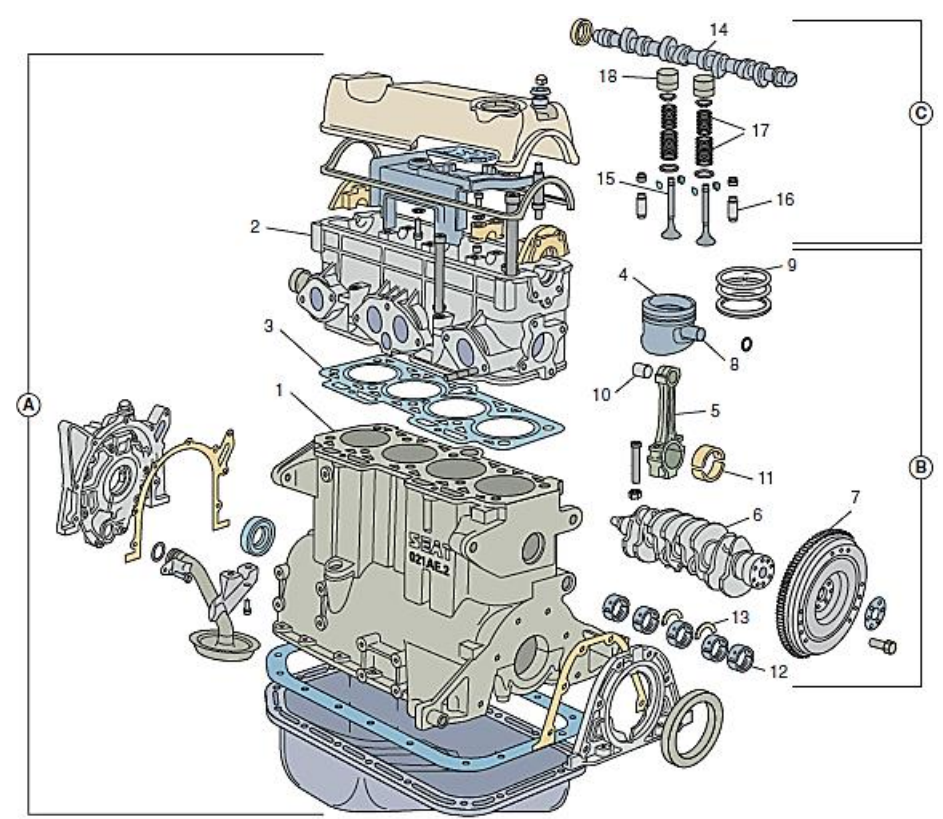

\begin{tabular}{|c|c|}
\hline $\begin{array}{c}\text { A. PARTES } \\
\text { ESTRUCTURALES }\end{array}$ & $\begin{array}{c}\text { B. TREN } \\
\text { ALTERNATIVO }\end{array}$ \\
\hline $\begin{array}{l}\text { 1. Bloque de } \\
\text { cilindros (block) }\end{array}$ & 4. Pistón \\
\hline 2.Culata (Cabezote) & 5. Biela \\
\hline \multirow[t]{8}{*}{ 3. Junta de culata } & 6. Cigüeñal \\
\hline & $\begin{array}{l}\text { 7. Volante de } \\
\text { inercia }\end{array}$ \\
\hline & 8. Bulón \\
\hline & 9. Segmentos \\
\hline & $\begin{array}{l}\text { 10. Casquillo de } \\
\text { biela }\end{array}$ \\
\hline & $\begin{array}{l}\text { 11. Cojinete de } \\
\text { biela }\end{array}$ \\
\hline & $\begin{array}{l}\text { 12. Cojinetes de } \\
\text { bancada }\end{array}$ \\
\hline & $\begin{array}{l}\text { 13.Cojinetes } \\
\text { axiales }\end{array}$ \\
\hline
\end{tabular}

Figura 5. Partes de un motor Otto de cuatro tiempos (Sanz, 2007). 


\subsection{Función de las partes de un motor Otto de cuatro tiempos}

La Figura 6 muestra un esquema de las principales partes del motor Otto 4T, siendo el block de cilindros el elemento estructural de mayor importancia y peso. En el block se montan partes fijas y móviles. Entre algunas partes fijas se tiene la culata y cárter, mientras que el cigüeñal es la parte móvil más importante, teniendo en cuenta que el pistón es una parte móvil que interactúa con el cilindro del block mediante el mecanismo de conexión biela-cigüeñal.

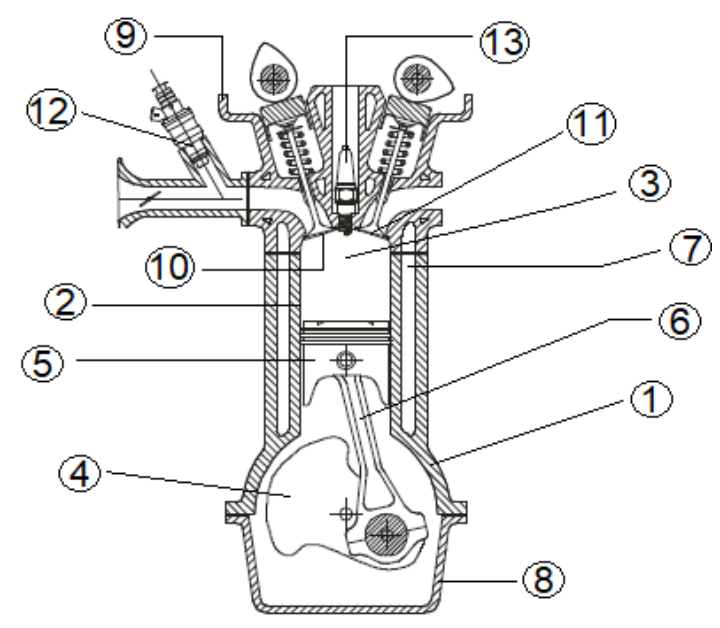

1 Block de cilindros
2 Cilindro
3 Cámara de Combustión
4 Cigüeñal
5 Pistón
6 Biela
7 Cámara de refrigeración
8 Cárter
9 Culata
10 Válvula de admisión
11 Válvula de escape
12 Inyector de combustible
13 Bujía

Figura 6. Esquema de las partes de un motor Otto de cuatro tiempos (Álvarez y Callejón, 2005).

Cada elemento de la Figura 6 tiene su función en el motor Otto de cuatro tiempos, por lo que, a continuación, se describe la función de cada una de ellas (Sanz, 2007) (Villegas et al., 2007) (Álvarez y Callejón, 2005).

- 1 Bloque: Denominado también como block, es un cuerpo estructural de mayor importancia en el motor donde se montan las partes móviles y fijas del mismo.

- 2 Cilindro: Habitáculo en donde se ubica y se guía el pistón, suele insertase una camisa de cilindro para evitar el desgaste.

- 3 Cámara de Combustión: Espacio físico real donde se introduce la mezcla airecombustible, para producir la combustión del combustible.

- 4 Cigüeñal: Recibe el impulso del pistón a través de la biela, lo que crea un par de fuerzas que se transforma en rotación.

- 5 Pistón: Recibe directamente la presión producida por la ignición del combustible, transmitiendo la fuerza a la biela.

- 6 Biela: Elemento de unión entre el pistón y cigüeñal, transmite al cigüeñal los esfuerzos recibidos por el pistón.

- 7 Cámara de refrigeración: Cavidades internas del block por donde circula el líquido refrigerante de los cilindros. 
- 8 Cárter: Cierre inferior del motor que contiene el aceite para la lubricación.

- 9 Culata: Denominada también como cabezote, cierra a los cilindros por la parte superior, y también contiene al sistema de distribución.

- 10 Válvula de admisión: Permite el ingreso de la mezcla aire-combustible.

- 11 Válvula de escape: Permite el escape de los gases de combustión.

- 12 Inyector de combustible: Inyecta el combustible a la cámara de combustión.

- 13 Bujía: Provoca la chista para iniciar el encendido de la mezcla airecombustible.

\subsection{Motor Otto de dos tiempos}

El motor Otto de dos tiempos posee cierta semejanza estructural que un motor Otto de cuatro tiempos. Las diferencias radican, en que, en vez de estar dotadas de válvulas de admisión y escape, los motores Otto $2 \mathrm{~T}$ poseen lumbreras de admisión, transferencia y escape, en el interior del block de cilindros. La Figura 7 muestra un esquema de las principales partes y lumbreras de un motor Otto de 2 tiempos.

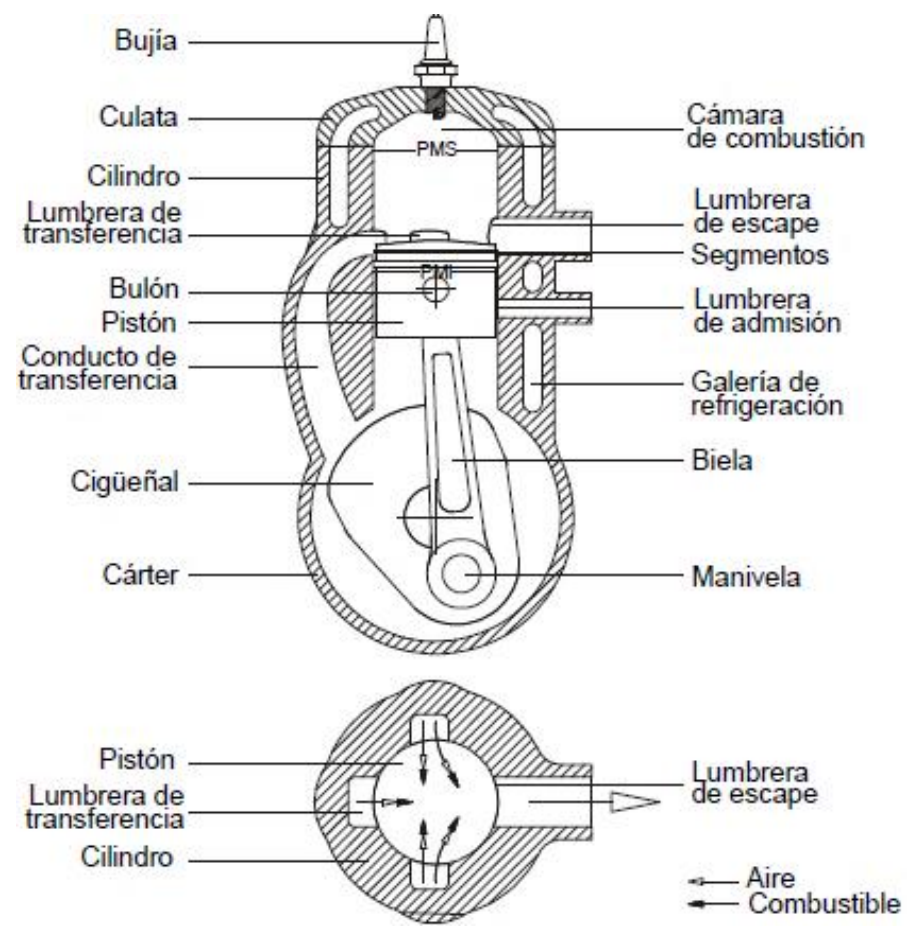

Figura 7. Esquema de las partes de un motor Otto de dos tiempos (Álvarez y Callejón, 2005).

Cada elemento de la Figura 7 tiene su función en el motor Otto 2T y los elementos en común con el motor Otto 4T realizan la misma función descrita anteriormente. A continuación, se describe la función de las lumbreras del block de cilindros de un motor Otto 2T. 
- Lumbrera de admisión: Cavidad interna del block por donde ingresa la mescla aire-combustible al cárter, debido al vacío que genera el pistón en su ascenso (Sanz, 2007) (Rovira y Muñoz, 2015).

- Lumbrera de transferencia: Cavidad interna del block que sirve para la transferencia de la mezcla aire-combustible, que se encuentra en el cárter, a la cámara de combustión del cilindro, esta transferencia se produce por la fuerza ejercida en el pistón en su descenso (Sanz, 2007) (Rovira y Muñoz, 2015).

- Lumbrera de escape: Cavidad interna del block por donde escapan los gases de combustión. (Sanz, 2007) (Rovira y Muñoz, 2015).

Dependiendo del diseño del motor Otto 2T pueden existir las tres lumbreras, pero en otros diseños solo dos lumbreras, lo cual depende del método de barrido de los gases de escape.

\subsection{Ciclo de trabajo de un motor Otto de cuatro tiempos}

El ciclo de trabajo de un motor Otto 4T está constituido por cuatro procesos o tiempos de trabajo: admisión, compresión, expansión y escape, como se muestra en la Figura 8. Para realizar el ciclo se requieren de cuatro carreras del pistón, en cada carrera del pistón el cigüeñal gira media vuelta, por tanto, el ciclo se completa en dos revoluciones del cigüeñal (Sanz, 2007) (Rovira y Muñoz, 2015). Los tiempos que se realizan en el ciclo de trabajo son los siguientes (Sanz, 2007) (Kaumar, 2019) (Kimerius Aicraft, 2011):

- Primer tiempo de admisión: El aire y el combustible, que se han mezclado proporcionalmente, entran por la válvula de admisión.

- Segundo tiempo de compresión: La mezcla aire-combustible es comprimida por el pistón y encendida mediante la chispa de una bujía.

- Tercer tiempo de expansión: La mezcla aire-combustible se deflagra y el pistón es presionado hacia abajo, la energía química de la mezcla se transforma en mecánica.

- Cuarto tiempo de escape: Los gases de escape, producto de la combustión, se conducen hacia fuera por medio de la válvula de escape.
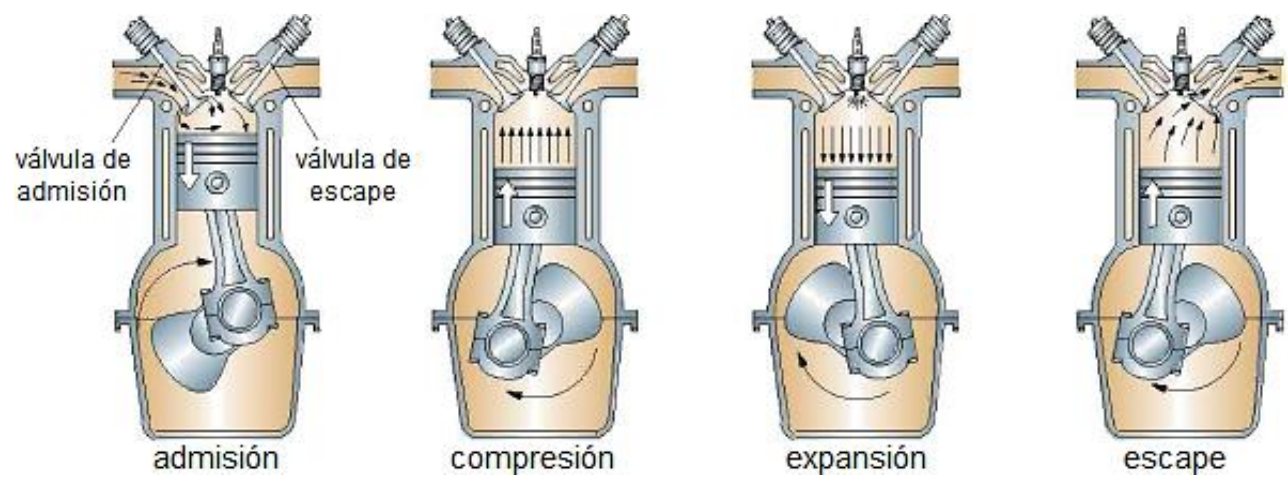

Figura 8. Ciclo de trabajo de un motor Otto de cuatro tiempos (Sanz, 2007). 


\subsection{Presión y temperatura en el cilindro del block de un motor Otto 4T}

El volumen variable que se genera entre cilindro-pistón-cabezote es el espacio donde se generan los procesos de admisión, compresión, expansión (combustión) y escape, además del espacio donde se desplaza linealmente el pistón. En este volumen se producen fenómenos químicos y físicos, debido a la combustión del combustible, que da como resultado el incremento de presión y temperatura al interior del cilindro.

El incremento de presión y temperatura, a causa de la ignición del combustible, ocasiona en la superficie de los cilindros del block, esfuerzo circunferencial, esfuerzo longitudinal, desgaste, vibración y corrosión, fenómenos por el cuales, los cilindros son fabricados con materiales que garanticen propiedades de resistencia mecánica, dureza, resistencia al desgaste, tenacidad, fatiga, bajo coeficiente de expansión térmica, alta conductividad térmica y anticorrosión, para evitar deformaciones, roturas y reblandecimiento del material. De estas propiedades las aleaciones de Al-Si cumplen con todas a excepción de bajo coeficiente de expansión térmica. En la Figura 9 se muestran las elevadas temperaturas y presiones, que soporta el cilindro del block de un motor Otto 4T durante su ciclo de trabajo.

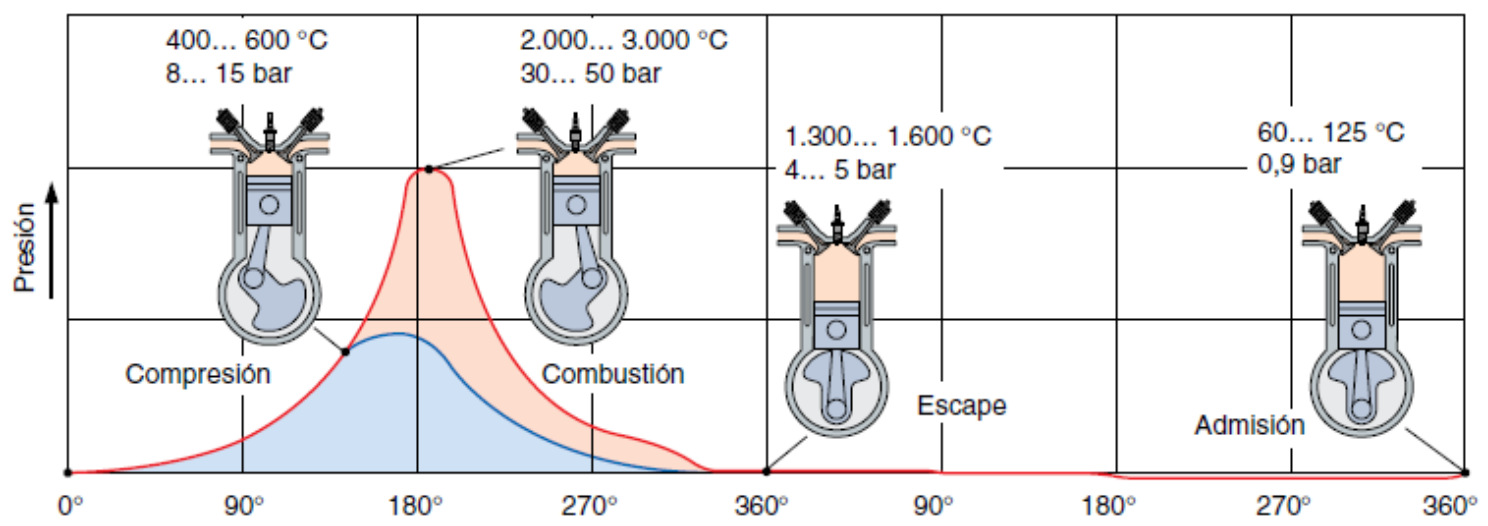

Figura 9. Temperaturas y presiones en el cilindro de un block de un motor Otto 4T (Sanz, 2007).

\subsection{Ciclo de trabajo de un motor Otto de dos tiempos}

El ciclo de trabajo de un motor Otto $2 \mathrm{~T}$ está constituido por dos procesos o tiempos de trabajo: compresión-admisión y expansión-escape. Para realizar el ciclo se requieren de dos carreras del pistón, en cada carrera del pistón el cigüeñal gira media vuelta, por tanto, el ciclo se completa en una revolución del cigüeñal (Sanz, 2007) (Rovira y Muñoz, 2015). Los tiempos que se realizan en el ciclo de trabajo son los siguientes:

- Primer tiempo compresión-admisión: El pistón asciende comprimiendo la mezcla de aire-combustible y simultáneamente crea un vacío en el cárter para que inicie el ingreso de la mezcla aire-combustible. La mezcla puede ingresar al cárter, debido a que el pistón al finalizar su carrera ascendente, deja libre la lumbrera de admisión y llena el cárter con la mezcla (Sanz, 2007) (Rovira y Muñoz, 2015) (Domínguez y Ferrer, 2019). 
- Segundo tiempo expansión-escape: La bujía provoca una chispa en el seno de la mezcla comprimida, creando una deflagración que empuja al pistón hacia abajo y precomprime la mezcla que se encuentra en el cárter, en su descenso deja libre la lumbrera de escape por donde salen los gases que son producto de la combustión. $\mathrm{Al}$ descender totalmente el pistón deja libre la lumbrera de transferencia (conecta cárter con el cilindro) por donde la mezcla aire-combustible ingresar nuevamente y ocupa el volumen del cilindro, y a la vez, el flujo de la mezcla, expulsa los últimos restos de los gases de escape, quedando preparado el cilindro para un nuevo ciclo de dos tiempos (Sanz, 2007) (Rovira y Muñoz, 2015) (Domínguez y Ferrer, 2019).

La Figura 10 muestra el ciclo de trabajo de un motor Otto de dos tiempos, en donde el ciclo ha dado inicio con el primer tiempo de compresión-admisión, para posteriormente finalizar con el segundo tiempo de expansión-escape.
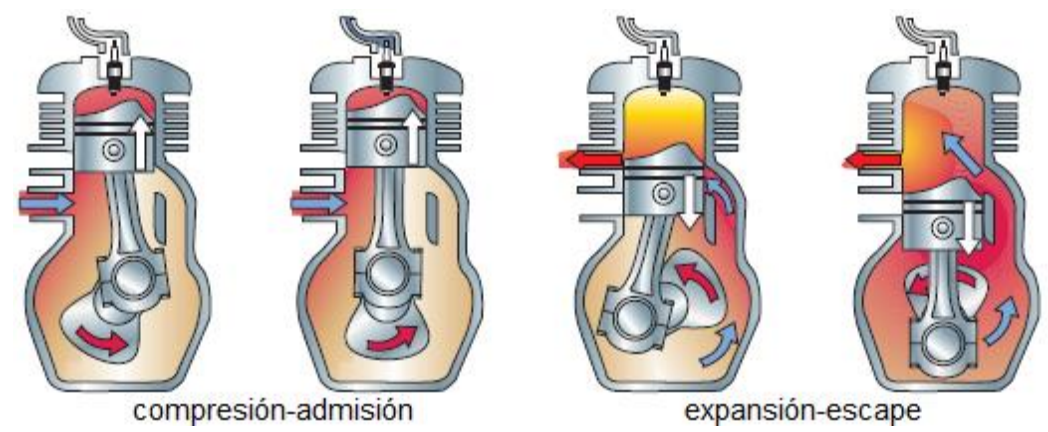

Figura 10. Ciclo de trabajo de un motor Otto de dos tiempos (Domínguez y Ferrer, 2019).

\subsection{Block de cilindros}

El block de cilindros es el elemento de mayor importancia de un motor, sobre el se montan otros componentes tanto fijos como móviles. Sobre su parte superior se asienta la culata con interposición de la junta de culata, consiguiendo la estanqueidad entre ambas partes. En la parte inferior se encuentra la bancada de cojinetes (semicárter del cigüeñal) donde se aloja el cigüeñal. La refrigeración del block de cilindros puede ser mediante liquido o aire (Sanz, 2007) (Gonzales, 2015).

El block de cilindros es un elemento macizo, el cual posee orificios internos llamados cilindros. Estos cilindros pueden ser parte integral del block, o piezas desmontables que se introducen al interior del block llamadas camisas. Las camisas son cilindros desmontables que se insertan en unos taladros que se han practicado en el block. La ventaja de utilizar camisas, radica en que el block se puede fabricar con materiales ligeros, y al mismo tiempo utilizar un material más denso y resistente para la fabricación de la camisa (Álvarez y Callejón, 2005) (Barrionuevo, 2013).

Geométricamente los cilindros deben ser estancos, perfectamente cilíndricos, de diámetro constante, sin ovalaciones, ni conicidades, y su interior debe presentar una elevada resistencia al desgaste, ya que en su superficie existe un desplazamiento continuo del pistón debido al ciclo de trabajo del motor (Álvarez y Callejón, 2005) (Barrionuevo, 2013). 
Como se mencionó, el block de cilindros puede ser fabricado como un solo elemento estructural o como un elemento más la inserción de camisas desmontables. En el primer caso, al no poseer camisas se tiene un block integral en el que sus cilindros pueden ser recubiertos con materiales resistentes al desgaste o realizar un tratamiento. En el segundo caso, se tiene un block con camisas húmedas o secas. Estos casos son algunas formas de clasificar al block de cilindros (Álvarez y Callejón, 2005) (Van Basshuysen y Schäfer, 2004). La Figura 11 muestra un block integral, y un block con camisas húmedas y camisas secas.
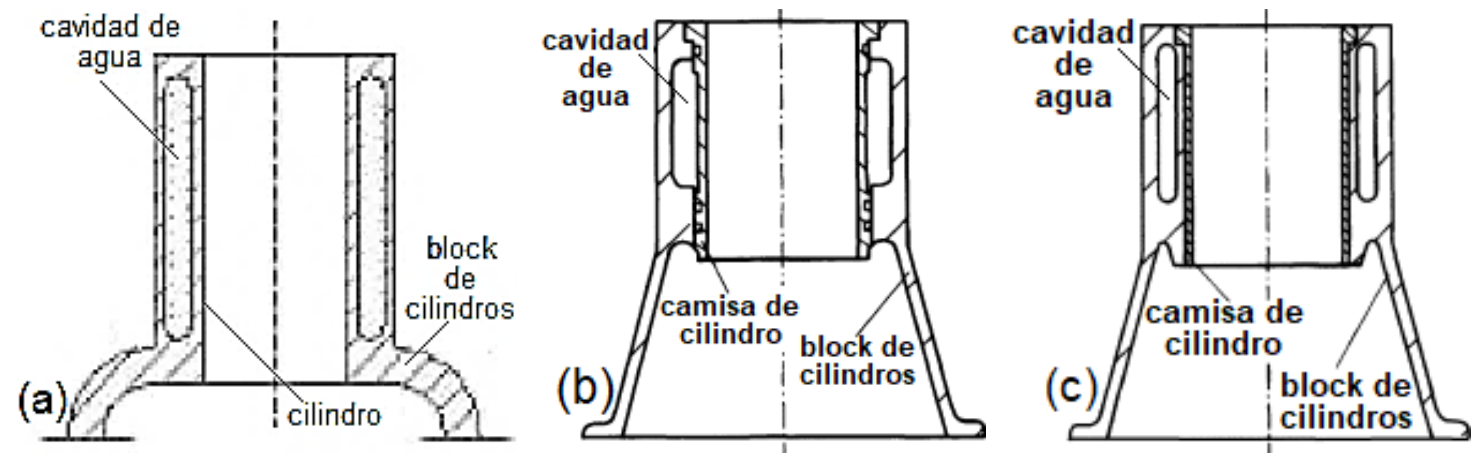

Figura 11. Block (a) integral, (b) con camisas húmedas y (c) con camisas secas (Álvarez y Callejón, 2005) (Van Basshuysen y Schäfer, 2004).

Tradicionalmente los blocks de cilindros de los motores Otto se fabricaban a partir de materiales de alta densidad como fundiciones grises y hierros dúctiles. Sin embargo, con el desarrollo a nivel industrial del aluminio, que fue desde el año de 1886, se pudo fabricar a mediados de 1950, un block de cilindros de aleación ligera de Al-Si, el cual consumía menos combustible. Actualmente los blocks de cilindros para motores Otto son fabricados con aleaciones de Al-Si, pero aún, este tipo de motores utilizan materiales de alta densidad.

\subsection{Partes estructurales de los MCIA tipo Otto fabricadas con aleaciones de Al-Si}

Actualmente partes estructurales como blocks de cilindros, camisas de cilindros, cabezotes, cárteres, bancadas de cojinetes y pistones, para motores Otto 4T de baja y mediana potencia, son fabricadas con aleaciones ligeras de Al-Si hipoeutéctico o hipereutéctico, ya que por propiedades como baja densidad, alta resistencia mecánica, dureza, resistencia al desgaste, tenacidad, resistencia a la fatiga, ductilidad, elevada conductividad térmica y anticorrosión, se logra un motor eficiente que tiene un menor consumo de combustible respecto a materiales de alta densidad.

En cambio, si las partes estructurales del motor son fabricadas a partir de fundiciones grises y hierros dúctiles, el rendimiento del motor se ve afectado por la alta densidad de estos materiales. Aun así, estas aleaciones de hierro-carbono, son utilizadas en vehículos de alto torque y potencia, debido a que tienen mayor resistencia que las aleaciones de aluminio-silicio. 
A continuación, la Figura 12, 13, 14, 15, 16, 17, 18 y 19, muestran las partes estructurales de los MCIA fabricados a partir de aleaciones de Al-Si.

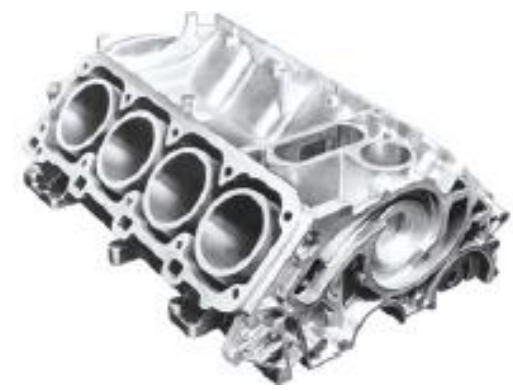

Figura 12. Block de cilindros de aleación Al-Si (MAHLE GmbH, 2016).

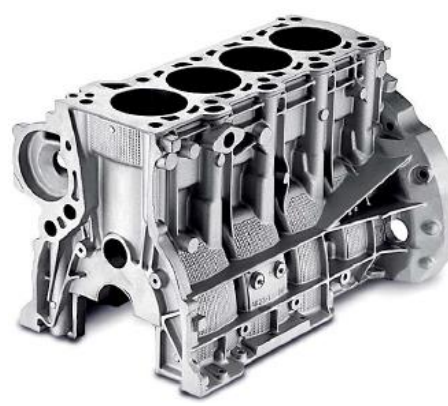

Figura 14. Block de cilindros de aleación Al-Si (Schilling y Schnaibel, 2009).

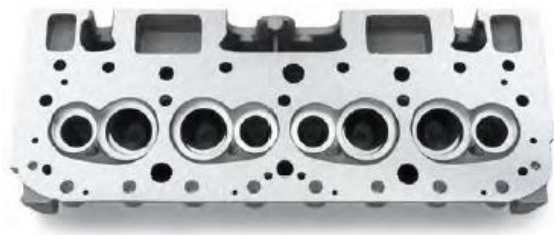

Figura 16. Cabezote de aleación Al-Si (General Motors, 2015).

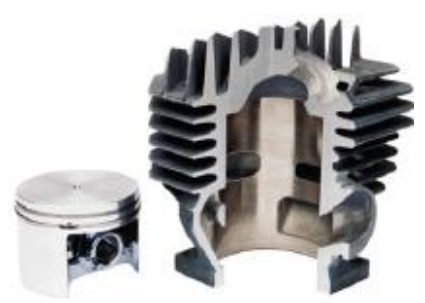

Figura 18. Cilindro y pistón de Al-Si (MAHLE $\mathrm{GmbH}, 2016$ ).

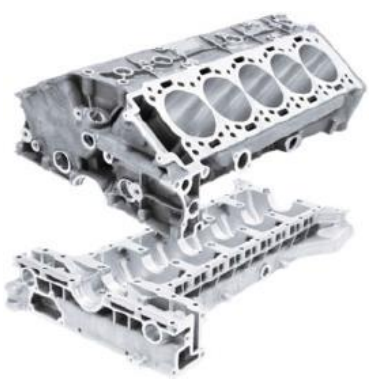

Figura 13. Block de cilindros con bancada de cojinetes de aleación Al-Si (MAHLE GmbH, 2016).

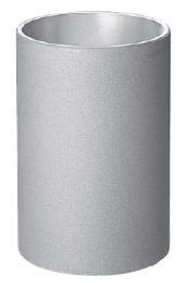

Figura 15. Camisa de cilindro de aleación Al-Si marca Silitec (Schilling y Schnaibel, 2009).

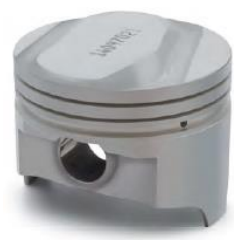

Figura 17. Pistón de aleación Al-Si (General Motors, 2015).

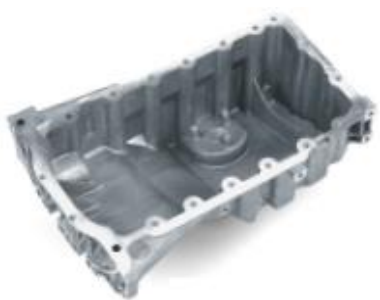

Figura 19. Cárter de Al-MgSi (Rheinfelden alloys, 2016). 


\section{MOTOR DIÉSEL}

Las características del motor Diésel radican en que su energía mecánica lo obtiene a partir de combustibles volátiles y viscosos, la ignición del combustible es por su inyección en el aire comprimido, y sus partes estructurales son robustas comparadas con los motores Otto. Según su ciclo de trabajo se clasifican en motores Diésel de cuatro tiempos (4T) y motores Diésel de dos tiempos (2T). La función de las partes principales de un motor Diésel 4T es semejante a los de un motor Diésel 2T, existiendo diferencias en sus ciclos de trabajo. El block de cilindros es el elemento de mayor importancia, sobre el, se montan otros componentes tanto fijos y móviles, posee orificios internos llamados cilindros que por lo general son piezas desmontables que se introducen en su interior llamadas camisas. Las partes estructurales de MCIA tipo Diésel son fabricadas de fundiciones grises y hierros dúctiles, ya que son motores con elevado torque-potencia.

\subsection{Características del motor diésel}

Reciben el nombre de motores de encendido por compresión, generalmente utiliza como combustible un aceite pesado comúnmente llamado gasóleo, pero también fuelóleo, aceites vegetales o biodiesel (Rovira y Muñoz, 2015) (Álvarez y Callejón, 2005).

En este tipo de motores el aire es comprimido al interior del cilindro del block (cámara de combustión). Finalizado el proceso de compresión se produce la inyección de combustible, originando la ignición (encendido), debido a la autoinflamación del combustible, a causa de la elevada presión del aire. Una vez iniciado la ignición del combustible sigue quemándose, mediante una combustión por difusión. Las condiciones que se deben alcanzar en el proceso de compresión serán aquellas que aseguren la autoinflamación de la mezcla (Rovira y Muñoz, 2015) (Domínguez y Ferrer, 2019).

Entre las diferencias con el motor Otto se encuentran que el motor diésel no requiere de una chispa de la bujía para la ignición del combustible, su encendido lo realiza por compresión de aire y posterior inyección de combustible. Además, el motor diésel posee una mayor carrera, por ende, una mayor relación de compresión (Sanz, 2007) (Rovira y Muñoz, 2015).

El motor Diésel de cuatro tiempos (4T) es utilizado en camionetas, tractores, cosechadoras, autobuses, camiones, plantas de generación eléctrica, grupos electrógenos, buques portacontenedores y toda clase de vehículos de elevado torque y potencia, este tipo de motores generalmente son refrigerados mediante agua. En cambio, el motor Diésel de dos tiempos (2T) es utilizado en maquinaria agrícola, vehículos ferroviarios, tanques de guerra, propulsión marina, buques, entre otros, este tipo de motores son refrigerados por agua o aire (Sanz, 2007) (Kimerius Aicraft, 2011) (Kuiken, 2008).

\subsection{Motor Diésel de cuatro tiempos}

Las partes estructurales tanto de un motor Otto 4T como las de un motor Diésel 4T son las misma, conformándose por un block de cilindros, camisas de cilindros y cabezote. Entre los diferentes motores Diésel existe variación en su forma y geometría, según la potencia, aplicación y marca del vehículo. Por otro lado, las partes del tren alternativo como las de distribución son fabricadas para llegar a un mismo fin, pero según la marca 
y aplicación, existen diferentes diseños. La Figura 20 muestra las partes principales de un motor Diésel de cuatro tiempos.

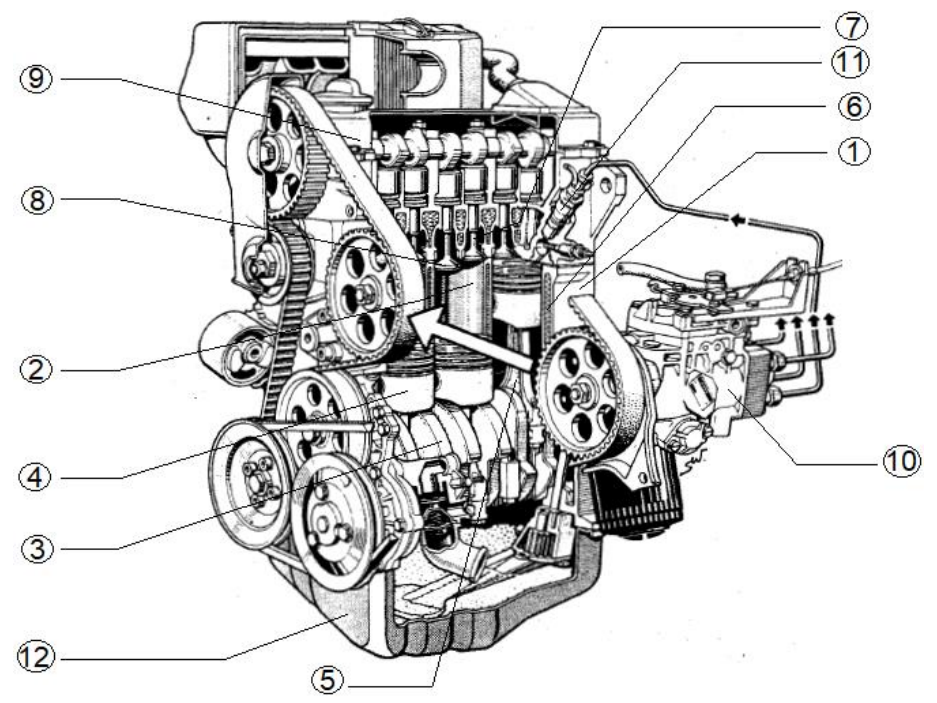

1 Block de cilindros
2 Cilindro
3 Cigüeñal
4 Pistón
5 Biela
6 Cámara de refrigeración
7 Válvula de admisión
8 Válvula de escape
9 Culata o cabezote
10 Bomba de inyección de combustible
11 Inyector de combustible
12 Cárter

Figura 20. Partes de un motor Diésel de cuatro tiempos (Heywood, 1988).

La Figura 21 muestra un esquema de las principales partes de un motor Diésel 4T, siendo el block de cilindros el elemento estructural de mayor importancia y peso del motor. Al igual que en el motor Otto, en el block de cilindros del motor Diésel se montan las partes fijas y móviles. Entre las partes fijas se tiene la culata y el cárter, mientras que el cigüeñal es la parte móvil más importante, teniendo en cuenta que el pistón es una parte móvil que interactúa con el cilindro del block mediante el mecanismo de conexión biela-cigüeñal.

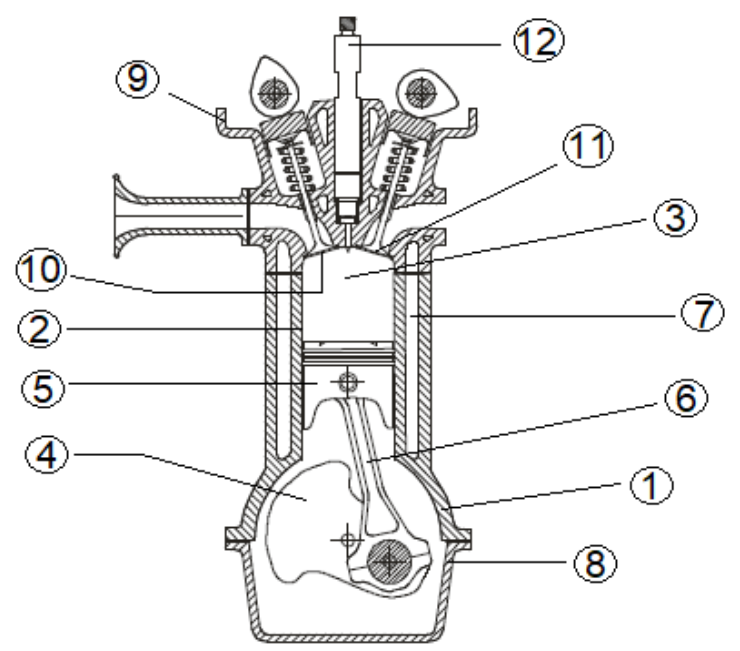
1 Block de cilindros
2 Cilindro
3 Cámara de Combustión
4 Cigüeñal
5 Pistón
6 Biela
7 Cámara de refrigeración
8 Cárter
9 Culata
10 Válvula de admisión
11 Válvula de escape
12 Inyector de combustible

Figura 21. Esquema de las partes de un motor Diesel de cuatro tiempos (Álvarez y Callejón, 2005).

El motor Diésel 4T esta implementado de un turbocompresor que logra aumentar la presión de aire atmosférico al momento de su ingreso en la cámara de combustión. Los principales objetivos de un turbocompresor es barrer los gases de escape y que el combustible inyectado reaccione por completo con el aire. 


\subsection{Motor Diésel de dos tiempos}

Un motor Diésel de dos tiempos posee cierta semejanza estructural que un motor Diésel de cuatro tiempos. Las diferencias radican, en que, en vez de estar dotadas de válvulas de admisión y escape, los motores Diésel $2 \mathrm{~T}$ poseen lumbreras de admisión, transferencia y escape, en el interior del block de cilindros. La Figura 22 muestra un esquema de las principales partes y lumbreras de un motor Diésel de 2 tiempos.

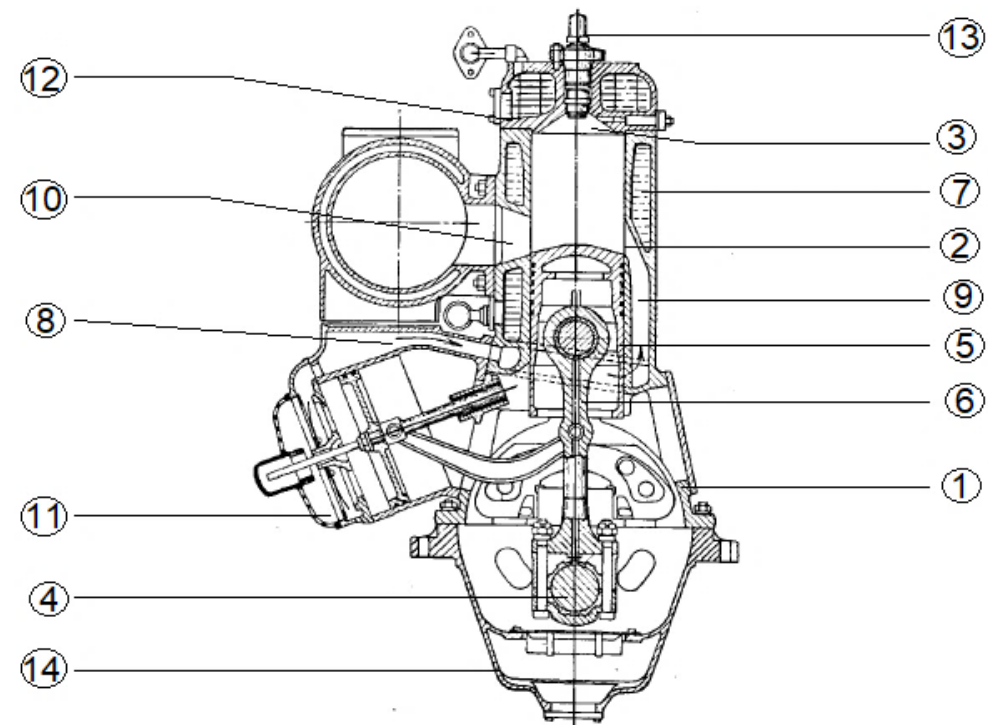

1 Block de cilindros
2 Cilindro
3 Cámara de combustión
4 Cigüeñal
5 Pistón
6 Biela
7 Cámara de refrigeración
8 Lumbrera de admisión
9 Lumbrera de transferencia
10 Lumbrera de escape
11 Blower
12 Culata
13 Bujía
14 Cárter

Figura 22. Esquema de las partes de un motor Otto de dos tiempos (Schweitzer, 1949).

\subsection{Ciclo de trabajo de un motor Diésel de cuatro tiempos}

El ciclo de trabajo del motor Diésel $4 \mathrm{~T}$ está constituido por cuatro procesos o tiempos de trabajo: admisión, compresión, expansión y escape, como se muestra en la Figura 23. Para realizar el ciclo se requieren de cuatro carreras del pistón, en cada carrera del pistón el cigüeñal gira media vuelta, por tanto, el ciclo se completa en dos revoluciones del cigüeñal (Sanz, 2007) (Rovira y Muñoz, 2015). Los tiempos que se realizan en el ciclo de trabajo son los siguientes (Sanz, 2007) (Villegas et al., 2007) (Domínguez y Ferrer, 2019):

- Primer tiempo de admisión: El aire comprimido por el turbocompresor entra por la válvula de admisión.

- Segundo tiempo de compresión: El aire es comprimido por el pistón, y justo antes de finalizar la compresión se realiza la inyección del combustible.

- Tercer tiempo de expansión: La elevada presión del aire origina la ignición del combustible generando que el pistón sea presionado hacia abajo, la energía química de la mezcla se transforma en mecánica.

- Cuarto tiempo de escape: Los gases de escape, producto de la combustión, se conducen hacia fuera por medio de la válvula de escape. 

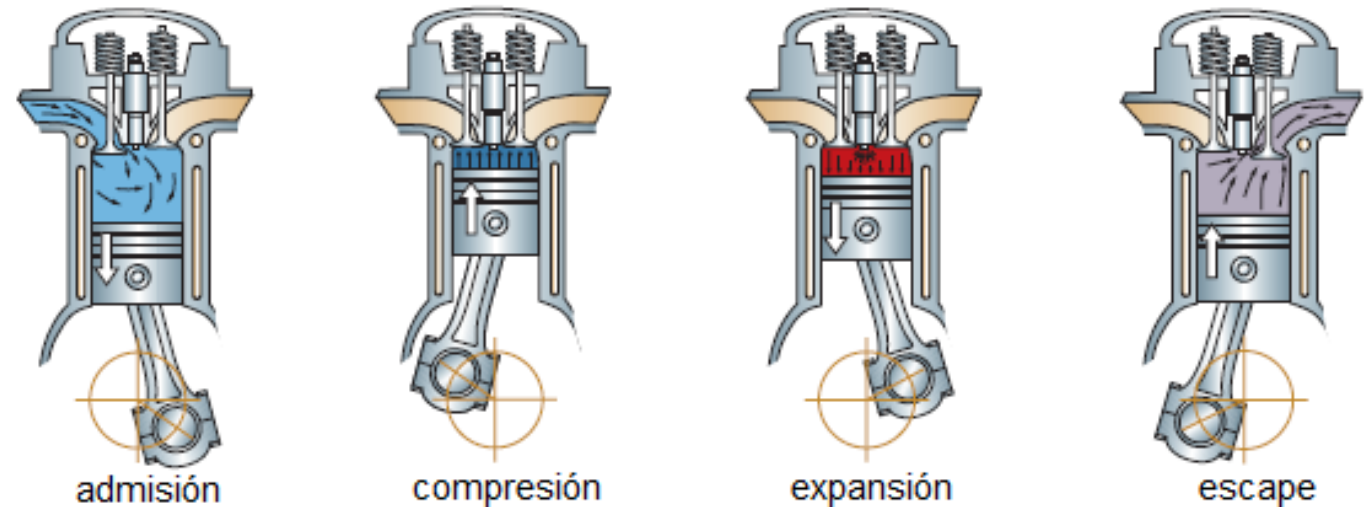

Figura 23. Ciclo de trabajo de un motor Diésel de cuatro tiempos (Domínguez y Ferrer, 2019).

\subsection{Ciclo de trabajo de un motor Diésel de dos tiempos}

El ciclo de trabajo de un motor Diésel 2T está constituido por dos procesos o tiempos de trabajo. Para realizar el ciclo se requieren de dos carreras del pistón, en cada carrera del pistón el cigüeñal gira media vuelta, por tanto, el ciclo se completa en una revolución del cigüeñal (Sanz, 2007) (Rovira y Muñoz, 2015). Los motores Diésel de dos tiempos pueden ser clasificados de acuerdo al modo de barrido de los gases (gases quemados son empujados por aire que entra al cilindro) y por el modo de alimentación del aire (atmosférico o sobrealimentado) (Gonzáles, 2015) (Arias, 1999). A continuación, se describirá el ciclo de trabajo de un motor Diésel de dos tiempos de acuerdo al modo de alimentación del aire.

\subsubsection{Ciclo de trabajo de un motor Diésel $2 \mathrm{~T}$ alimentado mediante aire atmosférico}

Los motores con este ciclo de trabajo son conocidos como motores atmosféricos, siendo de cilindrada baja. El pistón en su carrera ascendente crea una depresión que obliga la entrada de aire atmosférico en el cárter, seguidamente la presión que genera el pistón en su carrera descendente obliga al aire a pasar por la lumbrera de transferencia e ingresar al cilindro, lo que genera un barrido (evacuación) sobre los gases de combustión. Sin embargo, por ser aire con presión atmosférica, no es lo suficientemente fuerte para evacuar totalmente los gases de combustión. Normalmente este tipo de alimentación tiene muy poca aplicación en la industria (González, 2015) (Arias, 1999). A continuación, se describe el ciclo de trabajo de un motor Diésel 2T atmosférico.

- Primer tiempo compresión-admisión: El pistón en su carrera ascendente comprime el aire que se encuentra en el cilindro y simultáneamente crea un vacío en el cárter para permitir el ingreso de aire que se encuentra en la atmosfera, finalizada la carrera del pistón, también finaliza la entrada de aire al cárter (González, 2015) (Arias, 1999).

- Segundo tiempo expansión-escape: Finalizando el pistón su carrera ascendente de compresión, el inyector realiza la inyección de combustible sobre el aire comprimido que está a elevada presión, dando comienzo a la ignición del combustible, a causa de su autoinflamacion, lo que origina una presión y el descenso del pistón. Al ir descendiendo el pistón, en el cárter se genera una 
precompresión del aire que ha ingresado en el tiempo de admisión, y a la vez deja libre la lumbrera de escape por donde salen los gases combustionados. Finalizada la carrera descendente del pistón, este deja totalmente libre la lumbrera de carga, con el objetivo de que el aire precomprimido barra totalmente los gases combustionados e ingrese y ocupe nuevamente el volumen del cilindro, lo que da inicio a un nuevo ciclo de dos tiempos (González, 2015) (Arias, 1999) (Ferguson y Kirkpatrick, 2016).

La Figura 24 muestra el ciclo de trabajo de un motor Diésel 2T alimentado mediante aire atmosférico. Su ciclo de trabajo está constituido por dos procesos o tiempos de trabajo: compresión- admisión y expansión-escape.
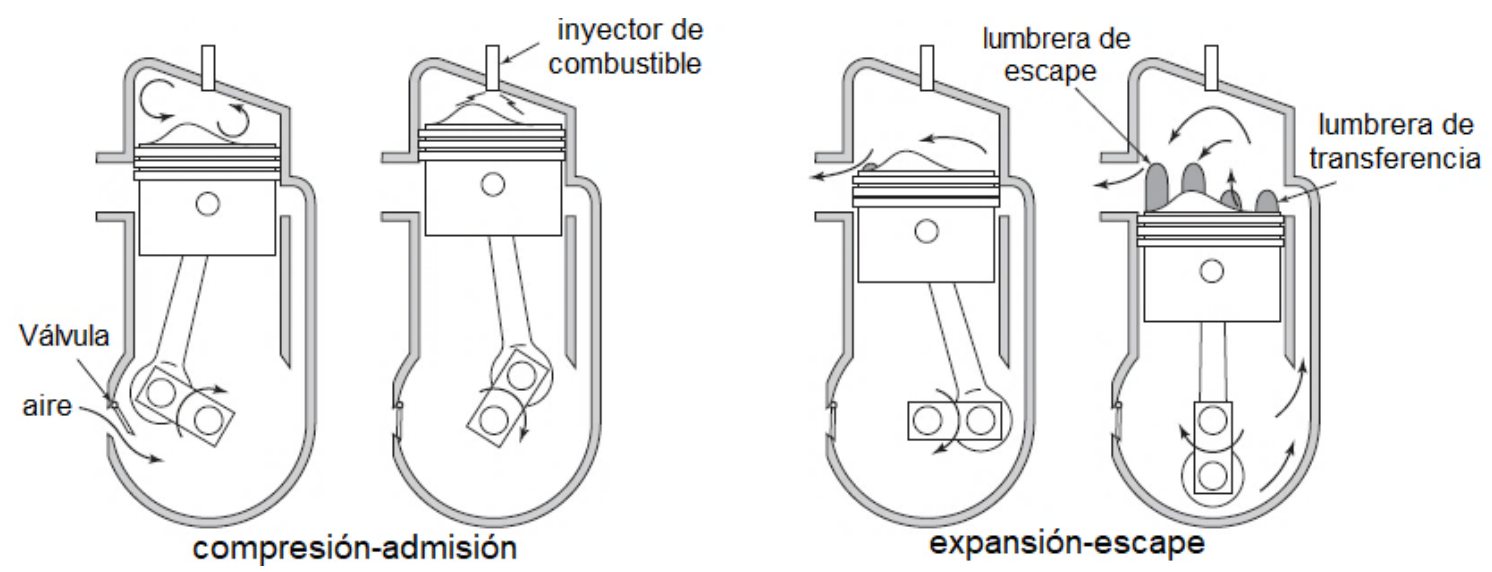

Figura 24. Ciclo de trabajo de un motor Diésel de dos tiempos alimentado mediante aire atmosférico (Ferguson y Kirkpatrick, 2016).

Este tipo de motores puede estar dotado de una bomba o blower, con el fin de aumentar ligeramente la presión del aire al momento ingresar al cárter. Además, el diseño del mecanismo para el ingreso del aire tiene varias formas.

\subsubsection{Ciclo de trabajo de un motor Diésel $2 \mathrm{~T}$ con sobrealimentación de aire}

Los motores con este ciclo de trabajo son conocidos como motores sobrealimentados, ya que el ingreso de aire en el cilindro se lo realiza mediante un compresor o turbocompresor. El aire que ingresa de esta forma tiene una presión elevada, que supera en gran medida a la presión atmosférica, siendo favorable para el barrido de los gases de combustión, ya que el ingreso de aire comprimido empuja a los gases combustionados al exterior. En este tipo de ciclos no existe lumbrera de escape, en su remplazo se tiene válvulas de escape, por lo que estos motores con sobrealimentación son extensamente utilizados en aplicaciones industriales (González, 2015) (Arias, 1999). A continuación, se describe el ciclo de trabajo de un motor Diésel 2T sobrealimentado.

- Primer tiempo escape-admisión: El pistón producto de la expansión se encuentra en su descenso y antes de abrir la lumbrera de admisión, se abren las válvulas de escape para permitir la salida de los gases de combustión. Una vez que el pistón ha descubierto la lumbrera de admisión el aire comprimido ingresa al cilindro y debido a su alta presión barre totalmente los gases de 
combustionados, instantáneamente se cierran las válvulas y el pistón comenzará su carrera ascendente de compresión (González, 2015) (Arias, 1999) (DOE Fundamentals Handbook, 1993).

- Segundo tiempo compresión-expansión: En el instante en que el pistón finaliza la carrera de compresión del aire, se realiza la inyección de combustible, causando la autoinflamación del mismo y de manera instantáneamente su combustión, la cual se expande a elevada presión y origina trabajo descendente en el pistón. Antes de que el pistón llegue totalmente a la parte inferior se abre las válvulas de escape para evacuar los gases de combustión y así comience un nuevo ciclo de trabajo (González, 2015) (Arias, 1999) (DOE Fundamentals Handbook, 1993).

La Figura 25 muestra el ciclo de trabajo de un motor Diésel 2T con sobrealimentación de aire, su ciclo de trabajo está constituido por dos procesos o tiempos de trabajo: escapeadmisión y compresión-expansión.
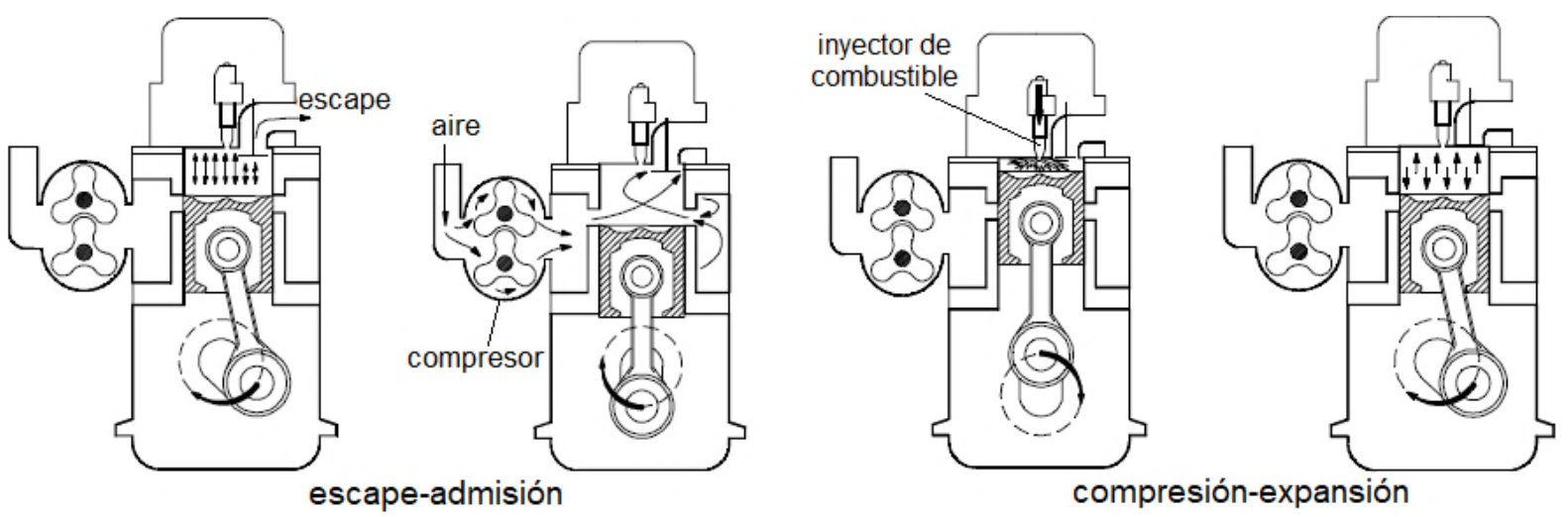

Figura 25. Ciclo de trabajo de un motor Diésel de dos tiempos con sobrealimentación de aire (DOE Fundamentals Handbook, 1993).

\section{RESUMEN DE CARACTERÍSTICAS DEL MOTOR DIÉSEL Y OTTO}

Las características de funcionamiento del motor Diésel y del motor Otto, tienen muchas semejanzas, sin embargo, existe diferencias notorias como en el tipo de combustible, relación de compresión, rendimiento efectivo, entre otras. De igual forma cada tipo de motor tiene sus ventajas y desventajas, lo cual permite establecer la aplicación en la industria.

\subsection{Características de funcionamiento del motor Diésel y del motor Otto}

Existen varias diferencias entre el motor Otto y el Diésel, desde los materiales estructurales utilizados para la fabricación de partes, hasta su rendimiento efectivo. Las principales características de funcionamiento de estos tipos de motores son presentadas en la Tabla 1. 
Tabla 1. Características de funcionamiento del motor Otto y del motor Diésel (Sanz, 2007)

\begin{tabular}{|c|c|}
\hline Motor Otto & Motor Diésel \\
\hline $\begin{array}{l}\text { Preparación de la mezcla en determinada } \\
\text { proporción. }\end{array}$ & Admisión de la máxima cantidad de aire. \\
\hline $\begin{array}{l}\text { En la admisión se regula la cantidad de mezcla } \\
\text { admitida. }\end{array}$ & $\begin{array}{l}\text { Alto grado de compresión (de } 14 \text { a 22/1), alcanzando } \\
\text { presiones de unos } 40 \text { bares. }\end{array}$ \\
\hline $\begin{array}{l}\text { Grado de compresión relativamente bajo, para } \\
\text { evitar la detonación (de } 8 \text { a 11/1), con presiones }\end{array}$ & 500 a $\left.600^{\circ} \mathrm{C}\right)$ \\
\hline entre 13 y 15 bares. & $\begin{array}{l}\text { Inyección de combustible en cantidad dosificada, a } \\
\text { una presión entre } 250 \text { y } 2000 \text { bares en los Diésel }\end{array}$ \\
\hline $\begin{array}{l}\text { El encendido de la mezcla se logra mediante una } \\
\text { chispa eléctrica. }\end{array}$ & rápidos de inyección directa. \\
\hline $\begin{array}{l}\text { La combustión es rápida y se realiza a volumen } \\
\text { casi constante. }\end{array}$ & $\begin{array}{l}\text { Autoinflamación del combustible al ser inyectado. } \\
\text { La combustión es relativamente lenta, entre } 20^{\circ} \text { y } 40^{\circ} \\
\text { de giro de cigüeñal. }\end{array}$ \\
\hline $\begin{array}{l}\text { Presión máxima de combustión de } 30 \text { a } 40 \text { bares. } \\
\text { El rendimiento efectivo de los motores es de } \\
\text { aproximadamente entre el } 25 \% \text { al } 30 \% \text {. }\end{array}$ & $\begin{array}{l}\text { Presión máxima de combustión entre } 70 \text { y } 90 \text { bares. } \\
\text { El rendimiento efectivo de los motores es de } \\
\text { aproximadamente entre el } 30 \% \text { al } 40 \% \text {. }\end{array}$ \\
\hline
\end{tabular}

\subsection{Ventajas y desventajas del motor Diésel respecto al motor Otto}

Tanto el motor Otto como el Diésel tiene sus ventajas y desventajas, por tal motivo cada tipo de motor es utilizado en algún tipo de aplicación industrial. Las principales ventajas y desventajas del motor Diésel respecto al motor Otto son presentadas en la Tabla 2.

Tabla 2. Principales ventajas y desventajas del motor Diésel respecto al motor Otto.

\begin{tabular}{|c|c|}
\hline Ventajas & Desventajas \\
\hline $\begin{array}{l}\text { Mayor rendimiento térmico debido a que trabaja con } \\
\text { temperaturas más elevadas. }\end{array}$ & $\begin{array}{l}\text { Mayor peso, más ruidoso y de funcionamiento más } \\
\text { brusco. }\end{array}$ \\
\hline $\begin{array}{l}\text { Motor con mayor rendimiento efectivo, puesto que se } \\
\text { aprovecha mejor la energía del combustible. }\end{array}$ & Más caro, por los ajustes que requiere su fabricación. \\
\hline $\begin{array}{l}\text { Menos contaminante, ya que la combustión es más } \\
\text { completa, por tanto, los gases de escape son menos } \\
\text { tóxicos. }\end{array}$ & $\begin{array}{l}\text { El arranque en frío presenta más dificultades. } \\
\text { Las partes estructurales como el block de cilindros, } \\
\text { camisas de cilindros y cabezote, no pueden ser de } \\
\text { materiales de baja densidad como las aleaciones de }\end{array}$ \\
\hline Mayor duración con menor costo de mantenimiento. & Al-Si. \\
\hline $\begin{array}{l}\text { La potencia puede ser aumentada mediante un } \\
\text { turbocargador. }\end{array}$ & $\begin{array}{l}\text { Las partes estructurales son fabricados con } \\
\text { materiales de alta densidad como fundición gris y } \\
\text { hierro dúctil, lo cual genera más peso al motor. }\end{array}$ \\
\hline $\begin{array}{l}\text { Generan elevado torque lo cual es empleado en motores } \\
\text { de camiones, volquetas, autobuses, tractores, } \\
\text { retroexcavadoras, plantas de generación eléctrica, } \\
\text { grupos electrógenos, buques portacontenedores, } \\
\text { vehículos ferroviarios, tanques de guerra, propulsión } \\
\text { marina, buques. }\end{array}$ & $\begin{array}{l}\text { Lo costos de fabricación del motor diésel tienden a } \\
\text { ser mayores que los del motor a gasolina, } \\
\text { principalmente por su construcción robusta, } \\
\text { necesaria para soportar las altas solicitaciones } \\
\text { mecánicas. }\end{array}$ \\
\hline
\end{tabular}

Fuente: Elaboración propia basada en (Sanz, 2007) (Kimerius Aicraft, 2011) (Kuiken, 2008) (Shell, 2020) 


\section{CONCLUSIONES}

- Las partes estructurales de un MCIA, fabricadas con aleaciones Al-Si, son utilizadas para motores Otto, ya que, la ignición del combustible genera fenómenos físicos y químicos que este tipo de materiales pueden soportar.

- Las partes estructurales de los MCIA tipo Diésel son fabricadas a partir de fundiciones grises o hierros dúctiles, esto se debe, a que, tales materiales pueden soportar los fenómenos físicos y químicos producidos por la autoinflamación del combustible, y por la elevada relación de compresiones en este tipo de motores.

- Las partes estructurales de los MCIA Otto están directamente relacionados con las aleaciones de $\mathrm{Al}-\mathrm{Si}$, estos materiales presentan propiedades que benefician al rendimiento del motor, ya que cada día se requiere de un elevado rendimiento a un bajo consumo de combustible.

- Con la estructuración del artículo se define conceptos, características, ciclos de trabajo y función de partes, los mismos que dan a conocer la importancia que tienen las aleaciones Al-Si en las diferentes partes del MCIA.

- Las ventajas que presentan las propiedades de las aleaciones de Al-Si como: baja densidad, alta resistencia mecánica, dureza, resistencia al desgaste, tenacidad, fatiga, ductilidad, elevada conductividad térmica y anticorrosivo, hacen que se tenga un gran potencial para la innovación.

- La selección de las aleaciones, sean de Al-Si o de Fe-C; para la fabricación de partes estructurales como blocks de cilindros, camisas de cilindros, cabezotes y pistones; vienen dadas en función del tipo de MCIA.

- Las propiedades que debe tener la parte de mayor importancia de un MCIA tipo Otto o Diésel son resistencia mecánica, dureza, resistencia al desgaste, tenacidad, resistencia a la fatiga, bajo coeficiente de expansión térmica, alta conductividad térmica y resistencia a la corrosión. Sin embargo, las cinco primeras propiedades son primordiales, mientras que las restantes puede suplirse de alguna otra manera.

- Las propiedades de las partes, juegan un papel fundamental en el rendimiento actual de los MCIA. Sin embargo, este rendimiento, también se debe al mejoramiento de los sistemas electrónicos de inyección de combustible.

\section{REFERENCIAS BIBLIOGRÁFICAS}

Álvarez, J., \& Callejón, I. (2005). Motores alternativos de combustión interna. Barcelona: Universitat Politecnica de Catalunya.

Arias, J. (1999). Proceso de barrido en un motor Diésel de dos tiempos sobrealimentado (Tesis Doctoral). UPM. Madrid. 
Barrionuevo, C. (2013). Introducción a las plantas de propulsión naval. Perú: Marina de Guerra del Perú.

DOE Fundamentals Handbook. (1993). Mechanical Science Diesel Engine Fundamentals. Washington,D.C: EG\&G Idaho.

Domínguez, E., \& Ferrer, J. (2019). Mecánica del vehículo. Madrid: Editex.

Ferguson, C., \& Kirkpatrick. (2016). Internal combustion engines applied thermosciences. United Kingdom: Wiley.

General Motors. (2015). Performance vehicles parts racing. Detroit: Chevy ${ }^{\mathrm{TM}} \mathrm{GM} \AA$. Rheinfelden alloys. (2016). Primary aluminium Casting alloys. Recuperado de http://rheinfelden-alloys.eu/wp-content/uploads/2017/01/Handbook-PrimaryAluminium-Casting-Alloys_RHEINFELDEN-ALLOYS_2016_EN.pdf

González, D. (2015). Motores térmicos y sus sistemas auxiliares. Madrid: Paraninfo.

Heywood, J. (1988). Internal combustion engine fundamentals. USA: McGraw-Hill.

Kimerius Aicraft. (2011). Motores de combustión interna. recuperado de https://www.kimerius.com/automóviles-7/motores/

Kuiken, K. (2008). Diesel Engines for ship propulsion and power plants from 0 to $100000 \mathrm{~kW}$. Netherlands: FME CWM.

Kumar, R. (2019). Reciprocating engine combustion diagnostics. Switzerland: Springer.

MAHLE GmbH. (2016). Cylinder components properties, applications, materials (2nd ed). Germany: Springer Vieweg.

NOSOLOINGENIERIA. (2013). Motorwagen Benz 1886: El primer automóvil del mundo. Recuperado de https://nosoloingenieria.com/motorwagen-benz-primerautomovil-mundo/

pressreader, (27 abr. 2015). Un abuelo muy joven Los 100 años de MAN. Recuperado de https://www.pressreader.com/spain/transporte-undial/20150427/282316793584965

Rovira, A., \& Muñoz, M. (2015). Motores de combustión interna. Madrid: UNED.

Sanz, S. (2007). Motores Mantenimiento de vehículos autopropulsados. Madrid: Editex.

Schilling, U., \& Schnaibel, S. Reacondicionamiento de bloques de motores de aluminio, 2009. Heilbronn: MS Motor Service International GmbH.

Schweitzer, P. (1949). Scavenging two stroke cycle diesel engines. New York: Macmillan.

Shell. El tutor de lubricación Shell Modulo 2 Motores. 
Van Basshuysen, R., \& Schäfer, F. (2004). Internal Combustion Engine Handbook Basics, Components, Systems and Perspectives. Germany: Vieweg Verlag.

Villegas, A., Montseny, I., \& Celoni, S. (2007). Motores de combustión interna. Cataluña: IES Baix Montseny. 


\section{PARA CITAR EL ARTÍCULO INDEXADO.}

Barona, G., \& Velasteguí, L. (2019). Materiales de aleación aluminio-silicio aplicados en la fabricación de partes de motores de combustión interna alternativos Parte I. Revista electrónica Ciencia Digital, 3(3.2), 302-313. https://doi.org/10.33262/cienciadigital.v3i3.2.758

El artículo que se publica es de exclusiva responsabilidad de los autores y no necesariamente reflejan el pensamiento de la Revista Ciencia Digital.

El articulo queda en propiedad de la revista y, por tanto, su publicación parcial y/o total en otro medio tiene que ser autorizado por el director de la Revista Ciencia Digital.
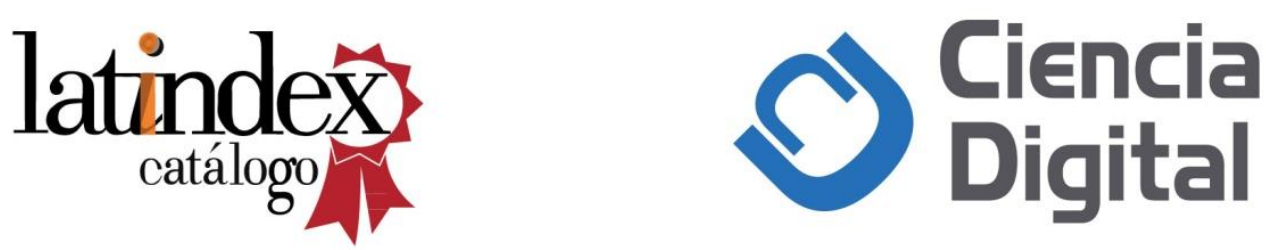